\title{
Paediatric Aspects of the Aberdeen Typhoid Outbreak
}

\author{
HUGH GALLOWAY, NORMAN S. CLARK, and MARGARET BLACKHALL \\ From the Department of Child Health, University of Aberdeen, Aberdeen City Hospital, \\ and the Royal Aberdeen Hospital for Sick Children
}

On May 16, 1964, a woman was admitted to Aberdeen City Hospital for Infectious Diseases for investigation of pyrexia. By May 20 the diagnosis of typhoid had been established in this patient and in several other patients admitted to the City Hospital and to Aberdeen Royal Infirmary between these two dates. From May 16 to July 31 when the last case was admitted, a total of 507 cases of typhoid was investigated and treated in Aberdeen hospitals, and 8 additional patients infected in Aberdeen are known to have been treated elsewhere-one of them as far away as Toronto. Of the 507 patients, 86 were children under the age of 12 years who were admitted between May 19 and June 25 . This paper gives an account of the clinical features and managemant of these 86 children, refers briefly to the bacteriological and serological findings in them, but does not touch at all upon the epidemiological or other public health aspects of the disease, which must obviously be considered with reference to the outbreak as a whole. As age does to some extent modify the clinical picture, we feel it may be of value to record separately our experience with this age-group in the first large epidemic of typhoid in this country for 30 years.

\section{Source}

The primary source of the epidemic was quickly identified as being the cooked meat counter of a supermarket in the main street of Aberdeen. There seems little doubt that the infection was introduced to this counter in a tin of corned beef and that it must have been rapidly and quite widely disseminated from the contents of this tin to other meats sold at the same counter, and probably to a very limited extent to other articles such as fruit sold on adjacent counters. The histories of 73 of the 86 children indicated that food bought at this supermarket had been consumed during the relevant period usually in their own homes or, occasionally, in the homes of near relatives. Pre-cooked meat of some variety

Received June 18, 1965. was included in the purchases in every case except one in which only fruit was involved. In a few cases there was some doubt as to whether the child had eaten the pre-cooked meat, but in one of these the mother volunteered that while she was waiting to be served at the meat counter, she found her child sucking the edge of the counter! Of the 13 children whose parents denied having shopped at this supermarket during the relevant period, 2 were almost certainly infected by sharing sweets with children already affected: another was possibly infected by a school meal prepared by a food handler who was herself in the early stages of typhoid: if this assumption is correct, it is fortunate and perhaps surprising that no other case could be traced to this source. In 10 children the source of infection could not be traced.

A striking feature of this outbreak was the social distribution of the cases. These children with few exceptions came from good homes, many of them from homes with high standards of hygiene and every material advantage. The explanation of this rather unusual distribution is very simple: bus routes serving all the most expensive housing areas in the city and many of the newest council housing estates at the periphery converge upon one end of the main street of Aberdeen at the front door of the supermarket concerned, and it is from these areas that the shop draws its large clientiele. Knowledge of this simple fact might have averted the publication of one of the more irrelevant of many misguided pronouncements in the press-an article in a reputable Sunday newspaper drawing attention to the inadequacies of housing in Aberdeen (based upon information which was 10 years out of date) and implying that this was playing an important part in the spread of the disease.

\section{Family Infections}

Family infections were common, as might be expected in view of the primary source of infection. This series of 86 children contains 13 pairs and one 


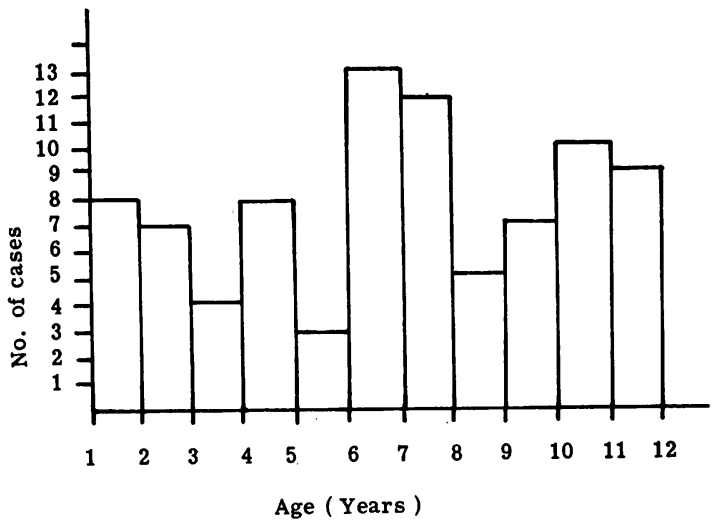

FIG. 1.-Age distribution of children admitted to hospital.

trio of sibs, and was therefore derived from 71 separate households. These households comprised 178 adults and 132 children (defined for this purpose as individuals below 15 years, the school leaving age). 61 of the 178 adults ( $34 \%$ ) and 92 of the 132 children $(70 \%)$ at risk were infected. The notably lower incidence in adults may be partly attributable to previous TAB immunization during military service, but we have unfortunately no information on their inoculation history. It seems likely that the difference in incidence rates reflects a higher susceptibility during childhood. Textbook opinions on this vary: according to Stuart and Pullen (1950) and Bradford (1964), the disease is commonest in young adults, while Watson (1958) states that it affects mainly children of school age.

\section{Clinical Features}

Sex and age incidence. The sex incidence was exactly equal, 43 boys and 43 girls. The age distribution of the 86 cases is shown in Fig. 1. The absence of any cases in babies under 1 year of age is presumably due to the nature of the source of infection, and it is clear that, given favourable circumstances for its dissemination, typhoid is not

TABLE I

Incubation Periods

\begin{tabular}{|c|c|c|c|c|c|c|c|}
\hline \multicolumn{7}{|c|}{ Incubation Period } & \multirow{2}{*}{$\begin{array}{c}\text { No. of Cases } \\
3 \\
25 \\
22 \\
4 \\
2 \\
30\end{array}$} \\
\hline $\begin{array}{l}<8 \text { days } \\
8-14 \text { days } \\
15-21 \text { days } \\
22-28 \text { days } \\
>28 \text { days } \\
\text { Uncertain }\end{array}$ & $\begin{array}{l}\cdots \\
\cdots \\
\cdots \\
\cdots\end{array}$ & $\begin{array}{l}\ldots \\
\cdots \\
\cdots \\
\cdots\end{array}$ & $\begin{array}{l}\cdots \\
\cdots \\
\cdots \\
\cdots \\
\cdots\end{array}$ & $\begin{array}{l}\cdots \\
\cdots \\
\cdots \\
\cdots \\
\cdots\end{array}$ & $\begin{array}{l}\cdots \\
\cdots \\
\cdots \\
\cdots\end{array}$ & $\begin{array}{l}\cdots \\
\cdots \\
\cdots \\
\cdots \\
\cdots\end{array}$ & \\
\hline Total .. & . & $\cdots$ & . & . & . & .. & 86 \\
\hline
\end{tabular}

'rare under the age of 2', as stated by Huckstep (1962).

Incubation period. Table I shows the incubation periods of 56 patients. The 30 patients whose incubation period remains uncertain include the 10 whose source of infection is unknown: in most of the remaining 20 , the uncertainty is due to the fact that food was bought from the infected shop on several occasions during the relevant period.

The shortest incubation period recorded was 5 days and the longest 34 days. The incubation periods in this case and in the other case recorded as over 28 days must be regarded as doubtful: both periods are calculated from dates on which they undoubtedly ate meat from the primary source, but each child was subsequently in contact with a proved case of the disease, and it is perhaps more likely that both were secondary cases with short incubation periods rather than primary cases.

TABLE II Main Symptoms in Order of Frequency

\begin{tabular}{|c|c|c|c|c|c|c|}
\hline \multicolumn{5}{|c|}{ Symptom } & Present & Absent \\
\hline $\begin{array}{l}\text { Fever } \\
\text { Malaise } \\
\text { Anorexia } \\
\text { Vomiting } \\
\text { Headache } \\
\text { Abdominal } \\
\text { Diarrhoea } \\
\text { Cough } \\
\text { Constipatio } \\
\text { Sore throat } \\
\text { Epistaxis } \\
\text { Muscle pai }\end{array}$ & $\begin{array}{l}\cdots \\
\cdots \\
\cdots \\
\text { pain } \\
\therefore \\
\text { on } \\
\text { ins }\end{array}$ & $\begin{array}{c}\ldots \\
\ldots \\
\cdots \\
\text { or } \mathrm{d} \\
\ldots \\
\ldots \\
\ldots \\
\ldots \\
\ldots\end{array}$ & $\begin{array}{l}\ldots \\
\ldots \\
\ldots \\
\ddot{\text { omfort }} \\
\ldots \\
\ldots \\
\ldots \\
\ldots \\
\ldots\end{array}$ & $\begin{array}{l}\cdots \\
\cdots \\
\cdots \\
\cdots \\
\cdots \\
\cdots \\
\cdots \\
\cdots \\
\cdots\end{array}$ & $\begin{array}{r}72 \\
58 \\
57 \\
42 \\
40 \\
31 \\
31 \\
25 \\
21 \\
16 \\
13 \\
9\end{array}$ & $\begin{array}{r}5 \\
3 \\
6 \\
20 \\
27 \\
26 \\
35 \\
11 \\
21 \\
6 \\
34 \\
4\end{array}$ \\
\hline
\end{tabular}

Symptoms. In Table II are listed the main symptoms in order of frequency. The number of negative as well as positive histories for each symptom is given in this Table, as each symptom is not mentioned specifically in every case history. The histories were taken by a number of different house physicians, at times working under considerable pressure, and it is not surprising that some of the records are less than perfect.

It was clear from the histories that, as always, typhoid was presenting a very variable picture both in severity and in the combinations of symptoms described. Only the non-specific symptoms of fever, malaise, and anorexia were positively mentioned in a majority of cases. 65 patients had a history of one or more of the triad - abdominal discomfort, constipation, diarrhoea. While some of the children had obviously been quite ill, others had few 
symptoms of trivial degree, and 2 were symptomless carriers discovered when tested as contacts of established cases. Even in the presence of an epidemic, early diagnosis is always difficult and often impossible. Only 12 of these children had symptoms of 3 days' duration or less on admission, while 40 had a history of 7 days or more. It was notable that short histories before admission were no commoner in the later stages of the epidemic when family doctors were more alert to the possibility of the diagnosis. Experience in this outbreak does not support the view of Watson (1958) that in children the onset tends to be more sudden than in adults.

Physical signs on admission. Physical signs were on the whole scanty and, with the exception of the rash, seldom made very helpful individual contributions towards the diagnosis, though the over-all picture was often suggestive in the moderately or severely ill. Rose spots, usually scanty, were present on admission or appeared in the next day or two in 37 children (43\%). When present they were the most valuable and indeed the only single diagnostic sign. The frequency of rose spots in white patients has varied in previous reports from $10 \%$ (Nelson and Pijper, 1951) to nearly 50\% (Marmion, 1952). The spleen was palpable in 27 children ( $31 \%$ ), but only in 1 or 2 cases was the tip more than one finger below the costal margin. The abdomen was noted as being tumid in 9 children on first admission. The records are incomplete on the frequency with which this sign subsequentiy developed, but it was seldom a very prominent feature. Vague complaints of ill-defined abdominal tenderness were common, but the degree and even the presence of significant tenderness were difficult to assess in children who were moderately ill and often upset by recent hospital admission. Mild signs of bronchitis were detected in only 4 children. To one of us (N.S.C.), who had considerable previous experience of typhoid in India, this was one of the most surprising features of the present outbreak, as past experience had suggested that respiratory signs were frequent and sometimes very prominent, and that the combination of respiratory and abdominal signs was often most useful diagnostically. Descriptions of typhoid based on experience in the tropics (Watson, 1958; Huckstep, 1962) support the view that signs of bronchitis are very frequent.

The general clinical impression of the severity of the illness on admission was as follows: severely ill 3 , moderately ill 42 , mildly ill 26 , not ill 15 . The children who did not appear ill had all been admitted following positive bacteriological tests carried out because they were home contacts of known cases. 2 of them had no signs or symptoms at any time, and in the remainder, symptoms had been minimal: 7 remained afebrile throughout, and 8 had brief low grade fever. The moderately and severely ill children were for the most part lethargic and mentally dull at first and many of them slept for long periods in the earlier stages of their illness. Delirium and mental confusion were not noted on admission or subsequently in any case.

Initial laboratory findings. Results of blood cultures taken before or on admission are recorded in 83 patients of whom 58 were positive and 25 negative. In 41 patients stool specimens collected before admission or within 24 hours thereafter were positive and 26 were negative. In one patient in whom the diagnosis was in doubt for some time, initial blood culture and three successive stool cultures were negative: her fourth stool culture and a blood culture taken at the same time were positive. In the 18 children for whom no pre-treatment stool culture results were available, the diagnosis was established on blood culture or clinical grounds, and treatment was started before a stool specimen became available. Only 10 children were negative both on blood and stool culture on admission, and in all of them the family history and/or strong clinical evidence supported the diagnosis. In 7 of these, typhoid bacilli were recovered on blood culture (3) and/or stool culture (5), after the completion of the first course of treatment. Typhoid bacilli were not cultured at any stage in the illness from 3 children: 2 of them had typhoid $H$ titres of $1 / 25$ and $1 / 50$ on admission: the third child's admission Widal results could not be traced, but when she was discharged her typhoid Vi titre was $1 / 20$. In view of the fact that in the great majority of cases only one stool was examined on admission, the isolation rate of typhoid bacilli ( 41 in 68 patients) was higher than is commonly reported in the early stages of the illness. Of the 41 positive stool specimens, 28 were passed in the first week of the illness, 3 of them within 48 hours of the first symptom.

Clinical course. The severity and duration of the illness varied as widely as did the initial signs. Generally speaking, as the temperature returned to normal, the symptoms disappeared and the child became quite lively, but a few remained obviously unwell for a few days after cessation of fever and others showed little evidence of general upset, though the temperature remained moderately raised. The duration of continuous fever varied from 24 hours to 22 days. One child who appeared perfectly well 
throughout showed intermittent low spikes of fever $\left(99-100^{\circ} \mathrm{F} .\left(37 \cdot 5^{\circ} \mathrm{C}\right.\right.$. $\left.)\right)$ for 5 weeks. Diarrhoea was a prominent feature in very few children, many passed one or two loose stools per day for a day or two in the second week, and constipation throughout was not uncommon. One child passed stools streaked with blood and mucus for almost two weeks. Abdominal discomfort in the earlier stages was common but significant abdominal pain was rare. The first child admitted, a boy aged 11 years, was the only one to develop a serious complication and the only one who was regarded as dangerously ill: on the seventh day of his illness he developed severe and repeated melaena for which he required two blood transfusions in the next 48 hours; thereafter his course was uneventful. Gross intestinal haemorrhage is generally considered to be a complication of the third week of typhoid, though it has been reported at any time from the end of the first week onwards. The great majority of the children were well and active by the time they were discharged from hospital, though a few remained listless and easily tired. 34 children (21 girls) shed a significant amount of hair during convalescence but only two showed a marked alopecia. This number included all 4 whose fever continued for 21 days or more, but this complication was also noted in children who were virtually afebrile throughout. In all of them re-growth of hair was satisfactory when they were seen 3 months after their discharge.

\section{Management and Treatment}

General management. One of the major problems in the general management of the outbreak was the provision of beds. When it became obvious that a large number of cases must be expected, Aberdeen City Hospital was cleared except for two cubicle wards which were retained for the management of other acute infections. Two wards in this hospital were set aside for children with typhoid and the first 40 cases were admitted to them. As soon as it appeared likely that it would not be possible to accommodate all cases in the City Hospital, admissions to the Royal Aberdeen Hospital for Sick Children were restricted to emergency cases, and two wards with a total capacity of 60 beds were cleared. The remaining 46 cases admitted from May 31 onwards were treated in these wards. Fig. 2 shows the daily number of children admitted. At no time was there any delay in the admission of proved or suspected cases.

Additional bed-pan sterilizers were provided in the sluices at the Children's Hospital and all bedpans and urinals were boiled immediately after use. The time-honoured practice of soaking stools in antiseptic before disposal was not employed: unless faecal matter is first homogenized with the antiseptic fluid, it is difficult to believe that soaking in antiseptic can achieve anything more than surface disinfection of solid faecal matter, and with satisfactory water-carriage sanitation immediate disposal appears the safer course. Bed linen was soaked in hypochlorite solution ('Milton', 1 in 80 ) immediately on removal, and spin-driers were provided in the sluices to remove as much fluid as possible before the linen was sent to the laundry.

Nursing care followed the generally accepted pattern, and in the almost complete absence of serious complications, presented no notable problems to nursing staffs already well accustomed to the technique of barrier nursing. No members of the medical, nursing, or other staffs were crossinfected. Dietary management was also on accepted lines, aiming at high calorie low roughage intake throughout the illness, with return to normal diet in the week or two before discharge. Except in the few children whose fever ran a prolonged course, anorexia presented no problem after the first few days. No visitors were allowed in the typhoid wards throughout the period of the outbreak as it was felt that in wards where children's hands and bed-clothes might well be contaminated from time to time, the supervision of a considerable number of

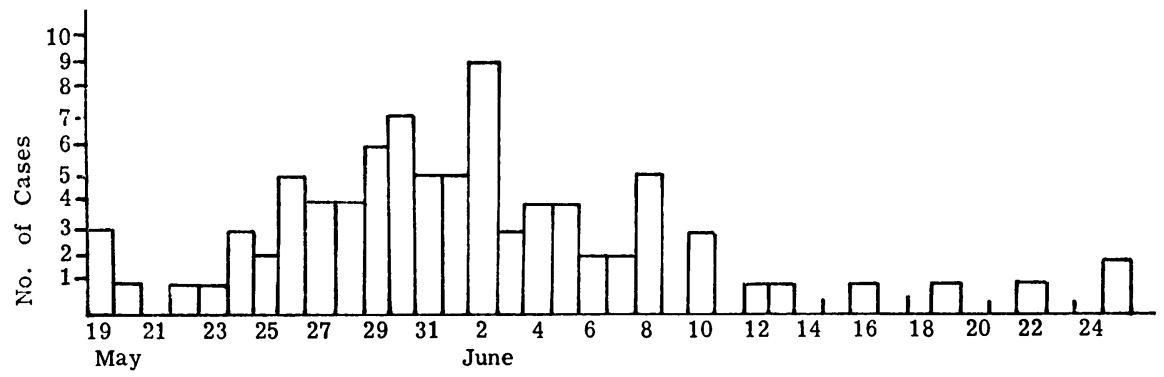

FIG. 2.-Daily number of admissions. 
visitors would place an impossible burden on an already hard-worked staff. Fortunately the construction of the wards, which were all at ground level, made 'window visiting' possible and this was freely permitted, beds being wheeled to the window.

Despite their prolonged stay in hospital and their lack of close contact with visitors, these children seem to have shown remarkably little evidence of psychological upset after their discharge home. Parents were questioned specifically about behaviour problems and other evidence of emotional upset when they returned with their children for a followup visit three months after discharge but, with a few exceptions among the younger children, emotional problems seem to have been brief or absent. While we would not for a moment advocate a return to the old pattern of visiting - or rather non-visiting-in children's hospitals, we would suggest that if the need really arises, visiting can be severely curtailed without serious or lasting psychological damage in the great majority of children.

One of the major problems was to keep the children, and particularly the older children, happy and occupied during their convalescence, while the results of clearance tests on stools and urine were awaited. In the case of children whose stools remained positive after the first course of treatment, this period of necessity commonly extended to 5 weeks or more. Here we were helped greatly by the Director of Education for the City of Aberdeen who arranged a supply of volunteer teachers who helped in the wards daily during the later stages of the epidemic by organizing handcrafts and other activities for the children.

Specific treatment. Chloramphenicol or ampicillin, or both drugs in sequence, were given in every case, the primary course of treatment lasting 14 days except in 5 cases in which the course was extended for various periods to a maximum of 25 days, because of fever continuing to 14 days or beyond. The earlier cases admitted to the City Hospital were all treated with chloramphenicol alone: a few of the later cases admitted to this hospital had ampicillin alone, and 13 of them had one week of chloramphenicol followed by one week of ampicillin. At the Children's Hospital, alternate cases were treated with chloramphenicol and ampicillin, but in a number of cases started on ampicillin treatment was changed to chloramphenicol because of failure of clinical response. In the whole series, 39 cases were treated with chloramphenicol alone, 24 with ampicillin alone, 13 with chloramphenicol for one week followed by ampicillin for one week, and 10 with ampicillin followed after a varying period by chloramphenicol. To simplify drug administration, it was decided to order dosage of drugs on an age basis rather than to relate dosage to weight. In retrospect, this decision was probably wrong: better results might have been achieved and assessment of results would certainly have been easier had dosage been ordered on a body weight basis. Chloramphenicol was given in a daily dosage of $1 \mathrm{~g}$. to all children aged 5 years and over, $750 \mathrm{mg}$. to children aged 3 and 4 years, and $500 \mathrm{mg}$. to children aged 1 and 2 years. Daily dosage of ampicillin was $1 \mathrm{~g}$. to all children except 1 - and 2-year-olds who received $500 \mathrm{mg}$., but these doses were doubled if clinical response was not satisfactory. Though the initial dose of both drugs in the majority of cases fell in the range $7-10 \mathrm{mg}$. $/ \mathrm{kg}$. day, the extremes were 4 and $14 \mathrm{mg}$. $/ \mathrm{kg}$. day.

In the dosage employed, chloramphenicol was clearly superior to ampicillin in the control of fever and early symptoms. In 52 cases treated initially with chloramphenicol, the average duration of fever after admission was 5.3 days, while in 34 cases treated initially with ampicillin, the average duration of fever was $10 \cdot 4$ days.

Stool and urine specimens from each child were cultured at 4-day intervals starting 4 days after completion of treatment. Children who were clinically well were discharged home when the first 3 stool and urine specimens were reported negative and 3 further specimens were examined at intervals after their return home. (This was a policy decision laid down for the epidemic as a whole.) No positive urine cultures were obtained from any child after treatment. All children who had a positive culture from any post-treatment stool specimen were retained in hospital and enrolled in a doubleblind clinical trial of ampicillin in larger dosage, $2 \mathrm{~g}$. daily for children under 6 years old and $3 \mathrm{~g}$. daily for older children, in each case for 10 days. This trial included adults and children and will not be further discussed as the results will be presented elsewhere.

While clinical response to treatment was satisfactory and, in chloramphenicol treated cases, moderately speedy, bacteriological results were disappointing and clinical relapses fairly common. Stool cultures were positive in 48 children ( $56 \%$ ), in 42 while they were still in hospital and in 6 after their discharge. Clinical relapse occurred in 12 of these 48 children and also in 6 children whose stools were never positive after the initial course of treatment (total clinical relapse rate $21 \%$ ). Of the 18 clinical relapses, 12 began during the second week after completion of the primary course of treatment. The shortest interval was 3 days and the longest 36 . Blood culture was positive in 11 cases during the 
relapse, but symptoms other than fever and lassitude were trivial or absent in all but 4 of these children. The majority of those with positive stools were already enrolled in the double-blind ampicillin trial before symptoms of relapse appeared, and only in one of these was it felt necessary to break the code in order to ensure that she should have active treatment. Five others were subsequently found to have been on control (inert) capsules or suspension but all had settled satisfactorily. Of the 6 children who relapsed with negative stools, 4 were treated with ampicillin in the same dosage as that used in the trial and 2 had no specific treatment. Table III shows the frequency of convalescent stool carriers and of clinical relapses following the four programmes of initial treatment. It will be seen that clinical relapse was much commoner after chloramphenicol than after ampicillin, but that the frequency of convalescent stool carriage was the same in both groups.

\section{TABLE III}

Results of Treatment

\begin{tabular}{|c|c|c|c|}
\hline & $\begin{array}{l}\text { Total } \\
\text { Cases }\end{array}$ & $\begin{array}{c}\text { Convales- } \\
\text { cent } \\
\text { Carriers }\end{array}$ & $\begin{array}{l}\text { Clinical } \\
\text { Relapse }\end{array}$ \\
\hline $\begin{array}{l}\text { Ampicillin } 14 \text { days } \ldots \\
\text { Chloramphenicol } 14 \text { days } \\
\text { Chloramphenicol } 7 \text { days; } \\
\text { ampicillin } 7 \text { days } \\
\text { Ampicillin/chloramphenicol }\end{array}$ & $\begin{array}{l}24 \\
39 \\
13 \\
10\end{array}$ & $\begin{array}{r}14 \\
21 \\
6 \\
7\end{array}$ & $\begin{array}{r}1 \\
12 \\
2 \\
3\end{array}$ \\
\hline & 86 & 48 & 18 \\
\hline
\end{tabular}

* Treatment was changed from ampicillin to chloramphenicol in this group because of poor response to the first drug: duration of treatment by each drug varied considerably from case to case.

A further course of ampicillin at home in dosage of $1 \mathrm{~g}$. per day for 3 months was given to 13 children whose stools were again positive after conclusion of the controlled ampicillin trial, and to 5 who produced positive stool cultures at home after discharge following initial treatment. All 86 children were seen again between 3 and 4 months after their discharge from hospital. All were completely recovered and showed no residual effects apart from some alopecia in a few. 3 stool and urine cultures carried out at weekly intervals were negative in all cases. (In those children who had 3-month courses of ampicillin, collection of stool and urine specimens was not begun till they had been off treatment for at least one week.) Further follow-up with stool and urine cultures at intervals will be carried out by the Public Health Department.

No serious side-effects of treatment were seen in any case. 2 children developed rashes while on treatment with chloramphenicol and 7 while on ampicillin. The rashes were mainly morbilliform in character and were not accompanied by constitutional upset or evidence of blood dyscrasia. In no case was treatment interrupted because of the rash.

\section{Summary}

Eighty-six children were affected in the 1964 Aberdeen typhoid outbreak.

The age and familial incidence and the clinical features of these cases are described and the difficulties of early diagnosis emphasized.

The disease generally ran a mild course and there were few complications and no deaths.

Chloramphenicol and ampicillin separately or in combination were given to all cases. Chloramphenicol was superior to ampicillin in the control of fever and early symptoms, but the clinical relapse rate was lower in those cases treated with ampicillin. Both drugs proved unsatisfactory in elimininating the organism from the stools: 48 children had positive stool cultures after conclusion of the first course of treatment.

\section{REFERENCES}

Bradford, W. L. (1964) Typhoid fever. In Textbook of Pediatrics, 8th ed., ed. W. E. Nelson, p. 488. Saunders, Philadelphia and London.

Huckstep, R. L. (1962). Typhoid Fever and Other Salmonella Infections. Livingstone, Edinburgh and London.

Marmion, D. E. (1952). The treatment of typhoid fever with chloramphenicol. Trans. roy. Soc. trop. Med. Hyg., 46, 619.

Nelson, H., and Pijper, A. (1951). Typhoid and paratyphoid fevers. In Modern Practice in Infectious Fevers, ed. H. S. Banks, Vol. 1, p. 349. Butterworth, London.

Stuart, B. M., and Pullen, R. L. (1950). Typhoid fever. In Communicable Diseases, ed. R. L. Pullen, p. 659. Kimpton, London.

Watson, K. C. (1958). Enteric fevers. In Diseases of Children in the Subtropics and Tropics, by H. C. Trowell and D. B. Jelliffe, p. 589 . Arnold, London. 